\title{
An Approach to Collaborative Learning \& the Serious Game Development
}

\author{
Corrigan, S. ${ }^{1}$, Zon, G. D. R. ${ }^{2}$, Maij, A. ${ }^{2}$, McDonald $^{1}$, N., \& Mårtensson, L. ${ }^{3}$ \\ ${ }^{1}$ Centre for Innovative Human Systems (CIHS), Trinity College, Dublin, Ireland \\ ${ }^{2}$ Dept of Training, Simulation and Operator Performance, National Aerospace Laboratory - NLR, Amsterdam, the \\ Netherlands \\ ${ }^{3}$ Dept of Industrial Economics and Management, KTH Royal Institute of Technology, Stockholm, Sweden \\ Corresponding author: S. Corrigan, siobhan.corrigan@tcd.ie, tel: +353 (0)1 6082605, fax: +353 (0)1 6712006
}

\begin{abstract}
This paper presents the overall learning process that evolved during the MASCA project (MAnaging System Change in Aviation - EU FP7 funded project $(2010$ - 2013), specifically focusing on the one of the key elements of the overall learning approach, the development of a Serious Game (SKYBOARD) and the role the game played in supporting the implementation of Airport Collaborative Decision Making (A-CDM) in a major European airport. The underlying principles of the learning process was based on on-going and collaborative learning in the workplace, with each phase of learning involving preparation and guidance, collaborative learning, consolidation of that learning and practically focused next steps that can be deployed to support overall change management. The aim of SKYBOARD was to aid communication and collaboration when introducing_A$\mathrm{CDM}$, and thereby supporting the cultural change that comes with this introduction. The development of SKYBOARD was based upon an initial training needs analysis and an iterative development and implementation approach at a major airport. The research demonstrated that we are at the beginning of a fundamental shift in the way both learning and working is happening in organisations. Therefore the establishment of a collaborative learning process and integrated learning package needs to focus on supporting continuous performance improvement and learning (competency and capability at all levels) and to ensure this overall learning is fully aligned to the overall strategic blueprint of the organisation. The evaluation of SKYBOARD demonstrated that Serious Games can support collaborative learning and enhanced communication and that such games should be key resource in any learning environment and proved to be a highly effective support to the implementation of A-CDM in this case.
\end{abstract}

Keywords: Collaborative Learning Process, Serious Games, SKYBOARD, Airport Collaborative Decision Making (A-CDM)

\section{INTRODUCTION}

The MASCA project was an action-based research project supporting the development, implementation \& assessment of change capability into aviation organisations responsible for and involved in change. The project focused on supporting change management case-studies across 2 European airports and 2 European airlines and the inclusion of a collaborative learning process was critical in order to achieve the right acquisition of skills and knowledge and facilitate the transfer on these skills into everyday operational practice. In MASCA an overall collaborative learning process was developed to support ongoing requirements as they emerged from the industrial change case studies within the project. The specific aim of this paper is to present the overall learning process with a specific focus on the role SKYBOARD (Serious Game) played in supporting one airport in their preparation of Airport Collaborative Decision Making (A-CDM). A-CDM is about partners (airport operators, aircraft operators/ground handlers, air traffic controllers and the Network Operations working together more efficiently and transparently in the way they work and share data.in order to improve the overall efficiency of operations at an airport, with a particular focus on the aircraft turn-round and pre-departure sequencing process. Therefore it requires a new focus of collaboration and learning across inter-agency organisations.

Traditionally the concept of 'learning' has been related to formal education, whereas its use in the context of work is a relatively new phenomenon. Interest in workplace learning has expanded since the beginning of the 1990s, and currently the research in this 
area is both wide-ranging and interdisciplinary. The reason for this expansion is the unprecedented rapid change in society and working life that has taken place during the past few decades. The rapid development of information and communications technology, the growing production of knowledge in the economy, increasing internationalisation and globalisation as well as changes in occupational structures all have contributed to a challenging and continuously changing working environment that requires personnel to be flexible and to quickly adapt to be able to operate in this environment. This work environment has made it necessary to find effective means of training that guarantee the preservation of high proficiency levels in employees (Tynjälä, 2008). The contents and organisation of work have challenged not only educational institutions but also work organisations to develop new ways of ensuring that the level of competency and capability of the workforce meets these challenges (Tynjälä, 2008). Thus, continuous and collaborative learning has become important both for individuals operating in the learning society and for organisations competing in international markets.

However many organisations are still focusing on the traditional approach to training. Figure 1 outlines 5 stages of work-place learning from stage 1 traditional classroom training right through to stage 5 more collaborative based training. The emerging evidence suggests that while many organisations are highlighting that they are not getting the value from stages $2-3$, this is still where the majority of training activities are focused. Hart (2012) found in her research that only $14 \%$ of organisations believe that the traditional approach to company training is an essential way for them to learn in the workplace.

To take the Aviation sector as an example, Human Factors training was recommended for all in the organisation from operational staff to senior management. The International Civil Aviation Organisation (ICAO) mandated Human Factors Training for various categories of aviation professionals. The European Aviation Safety Agency (EASA) also has requirements on Human Factors training. Furthermore the International Air Transport Association (IATA) requires Human Factors training through the two standards IATA Operational Safety Audit (IOSA) and IATA Safety Audit for Ground Operations (ISAGO). All the above requirements and standards resulted in a huge volume of Human Factors training offered to staff of Airlines, Aviation Maintenance Organisations, Airport Operators, Airport Handling Companies and Air Traffic Services.

But many years on from these recommendations- the question remains does this approach to training (again primarily falling between stages 1-3 as identified in figure 1) actually result in improved performance and enhanced capability in the workplace? Research from previous EU funded projects (ADAMS, Human Factors in Aircraft Dispatch and Maintenance, 1994-1999; ADAMS II, Human Factors in Aircraft Dispatch and Maintenance., 2001-2004; HILAS, 2005-2009) highlighted that during the Human Factors training programmes, frustrations were often expressed by staff that they were trying their best to apply the new learning but the system did not support them. Training was criticised by staff as being 'idealistic' and 'removed from the realities of the system'.

5 Stages of Workplace Learning (2010)

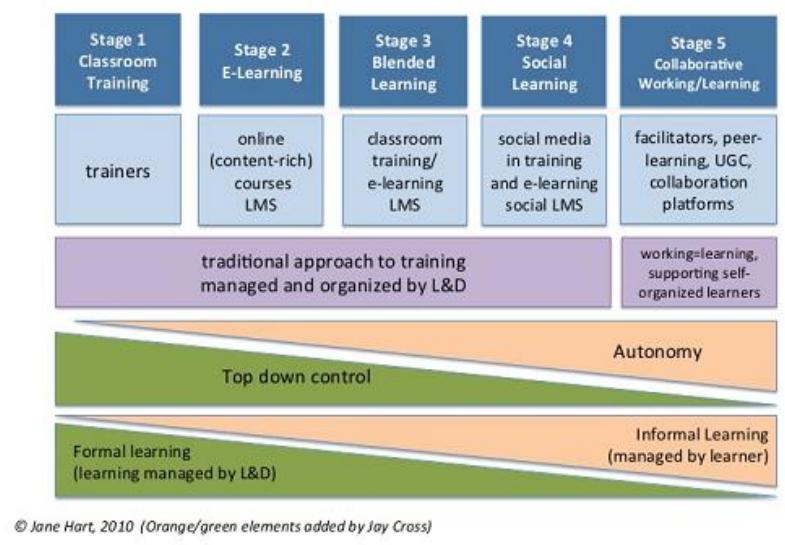

Fig 1 Five Stages of Current Work-Place Learning

Anecdotal evidence from aviation organisations suggests that it is difficult to demonstrate Return of Investment in terms of Human Factors training. They reported that they found formal training approaches to Human Factors "costly, repetitive, inconsistent and largely unmeasured" and they struggle to quantify the real value (increased safety and improved performance to ensure competitiveness) to their operations in the on-going investment in their training initiatives.

There is also evidence to suggest that as much as 60 to 80 per cent of the 'learning' that occurs in today's workplaces arises from the more informal, tacit and social systems of knowledge exchange and from what actually happens in normal operational practice 
(i.e., stage 5 learning) (ADAMS, Human Factors in Aircraft Dispatch and Maintenance, 1994-1999; HILAS, 2005-2009;

Johnsson, M., Boud, D., \& Solomon, N, 2012).This notion of on-going collaborative learning is a fundamental premise of the MASCA approach.

Collaborative learning is an approach based on the idea that learning is largely a social behaviour involving groups of learners working together as a team to find a solution and work together in implementing that solution. Collaboration is broadly defined as an interaction among two or more individuals and can encompass a variety of behaviours including communication, information sharing, co-operation, co-ordination, problem solving and negotiation in order to create an overall common and compatible operational picture.

One of the most important elements of learning which is absent from traditional approaches to training and learning is the process of social interaction in order to establish an understanding of this common and compatible process. This level of understanding and learning is vital for the success in sustaining change and ensuring on-going learning.

In order to successfully optimise the way this learning is developed and existing knowledge and experience is exploited, organisations need to facilitate the dynamic capabilities required for converting the knowledge available from the insights and competencies of people into appropriate structures, processes, products and systems that allow the value to be exploited. Therefore what the MASCA approach proposed was the requirement for a more flexible, operationally embedded deployment of on-going collaboration and learning opportunities within the air transport system - 'the right level of knowledge, to the right people at the right time'. Furthermore the knowledge and skill has to be based on a new understanding of how the complex system-of-systems of aviation works, how it should be managed, how it can be changed and how to design for learning and improved future system operations (MASCA, 2012).

\subsection{MASCA APPROACH TO COLLABORATIVE LEARNING}

The underlying theoretical principles of the MASCA learning approach was based on an on-going and collaborative learning process, with each phase involving preparation and guidance, collaborative learning, consolidation of that learning and practically focused next steps that can be deployed to enable overall change management (Mc Donald, 2012). Figure 2 describes a 5 phase approach to the overall MASCA learning process. This overall learning approach supported the key objective of the MASCA project in transferring change management capability into the organisations that are responsible for and involved in change.

\section{Phase 1: Understand the present}

Collaborative learning to build a common operational picture amongst diverse stakeholders in the operation begins with understanding each of the stakeholder's current working situations. It should start with understanding the 'local rationality' of each stakeholder group, making tacit knowledge explicit thus ensuring a full understanding of both formal and informal ways of working. This forms the basis for modelling the current as-is process or processes. Therefore the primary learning mode is assimilation of that new explicit information. This stage could be done separately for each stakeholder group to ensure that variants of the process are properly captured. When significant divergences between groups are expected, then it is not advisable to bring groups together prematurely, before building an explicit understanding of each local rationality (Mc Donald, 2012).

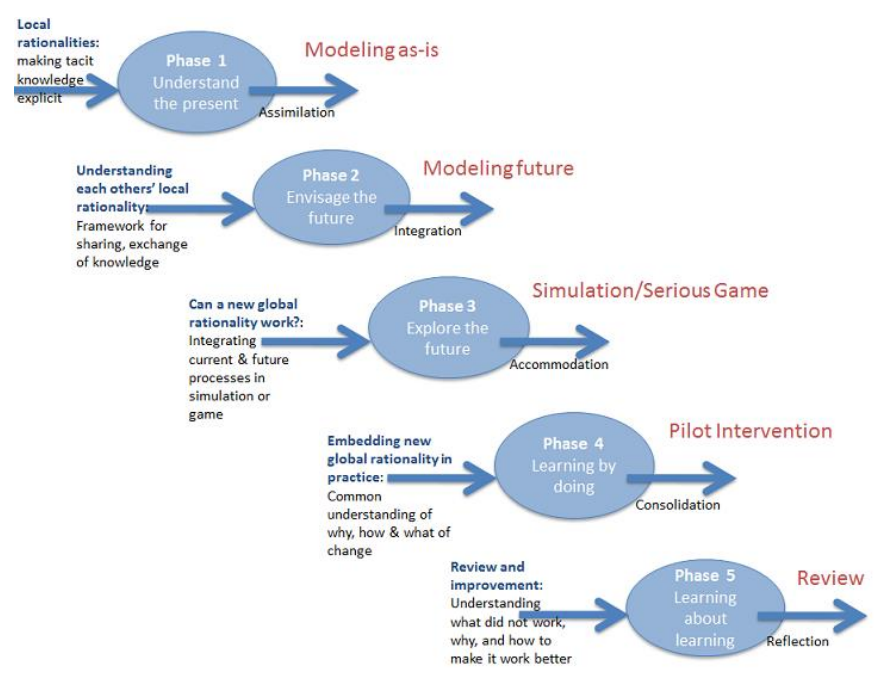


Fig 2 The MASCA Learning Process (Mc Donald, 2012)

Phase 2: Envisage the future.

The models of the as-is process(es) then provide a framework for sharing and exchanging information, enabling an understanding of each other's local rationalities. This provides the basis for a collaborative process of thinking of potential transformations of the as-is process into a future process - envisaging the future - leading to a model of the future process. The main learning mode here is integration of different models, making explicit the principles that enable the design of a future process.

\section{Phase 3: Explore the future.}

The functional adequacy of the future process model then needs to be tested in some kind of exploration. This can be a simulation or game (in this case it was the SKYBOARD Serious Game). This can be more or less formally constructed, depending on requirements and opportunities. The basic question is - can the new global rationality work? The main learning mode here is accommodation - the adjustment of understanding to the ways in which the future might work. The more active and engaging this exploration as in a serious game, the more it provides the opportunity to develop tacit knowledge generated through the actions and interactions of the game.

Phase 4: Learning by doing

The fourth stage of the learning model comprises the embedding of this new understanding (common operational picture or global rationality) into normal everyday practice, initially in a pilot trial or case study. This seeks to prioritise learning-by-doing to deepen and broaden practical understanding of the how, why and what of the changed reality. The primary learning mode is consolidation of all the learning of the previous stages into a new practice, based on shared understanding, intensifying the development of rich layers of tacit knowledge about how the system now works.

\section{Phase 5: Learning about learning}

The fifth stage involves reflection about how the change initiative worked (or not), generating a new improvement cycle. This provides a feedback loop to the plan and an opportunity for wide participation in learning. Middle management is critical in feed forward and feedback. This stage may provide direct opportunities for a higher level of collaboration or participation in the change, including locally-generated change initiatives. This stage is also critical at the strategic level and external change agents (e.g., research consortia, communities of practice etc) may be important in reviewing and learning lessons (Mc Donald, 2012).

\section{MASCA SERIOUS GAME (SKYBOARD)}

One of the key aspects of the overall MASCA learning process (phase 3) focused on the further exploration of a future process and new way of working. In his context the development of the SKYBOARD Serious Game provided an active and engaging medium to further explore how collaboration should work as part of the overall A-CDM change initiative. This section provides an overview of the development process, implementation and evaluation of the SKYBOARD Serious Game and how it supported the preparation for full implementation of A-CDM.

There are many different definitions of Serious Games and much discussion on what elements should be included in a serious game to distinguish it from other training media. One of the very first definitions stems from Abt (1970), who states that: "Serious Games have an explicit and carefully thought-out educational purpose and are not intended to be played primarily for amusement." Others are: "Serious game is a game in which education (in its various forms) is the primary goal, rather than entertainment" (Michael and Chen 2006, p.17). And: "Serious game: a mental contest, played with a computer in accordance with specific rules, that uses entertainment to further government or corporate training, education, health, public policy, and strategic communication objectives. “Zyda's (2005, p.26). Thus Serious Games are both educational and amusing, but their primary goal is not amusement. Serious games are often used in parallel with other learning tools and environments, such as lectures, e-learning forums, and simulators.

The interest in Serious Games from the scientific community has grown rapidly during the past ten years. More and more researchers are not only developing serious games, but are also investigating what makes a serious game an effective training and learning medium and how to ensure that players learn what they are intended to learn. These goals of Serious Games can vary from training competences to part-task training and from changing attitudes to creating awareness. However, not all serious games are equally effective in achieving their goals (e.g. Annetta, et al, 2009; Plass, et al., 2013). It depends on the game purpose and the type of game that is used how effective the game is. Also the ways to measure game effectiveness are not always sufficiently objective (Connolly, et al, 2012). Some games have proven to be effective while others are no more effective than 
other, less expensive, training media, such as literature and lectures (Coller \& Scott, 2009). The effectiveness of serious games can be partly contributed to what is intended to be taught using the game (Belanich, Orvis, \& Sibley, 2013). For example, typical knowledge elements may be better taught using literature and typical skill elements by using a simulator. The required elements that needed to be focussed on leaned heavily on skills and attitudes, which lend themselves for experiential learning (Kolb \& Kolb, 2005). Serious games may be specifically suited for this type of learning.

The advantage of using Serious Games over other learning tools is that serious games enhance participants' motivation (Garris, Ahlers, \& Driskell, 2002). In fact the games elicit them to play, and therefore learn, over and over again (Ritterfeld, Shen, Wang, Nocera, \& Ling Wong, 2009). A serious game is considered well-developed when the correct balance between entertainment and education is found. Therefore, the development of a serious game is a flexible process with many iterations and interactions between developers and potential users to ensure the correct balance and continuous and on-going learning. In the context of this paper a serious game (Skyboard) was developed, implemented and evaluated to support the implementation of Airport Collaborative Decision Making (A-CDM) in a large European airport. The objective of the serious game was to initiate a common understanding across the key stakeholder organisations and to highlight the level of collaboration and information sharing required in order to ensure the successful implementation of A-CDM at a major European airport.

\section{Methodology}

The development process of the SKYBOARD serious games was iterative in nature and is presented in figure 3.

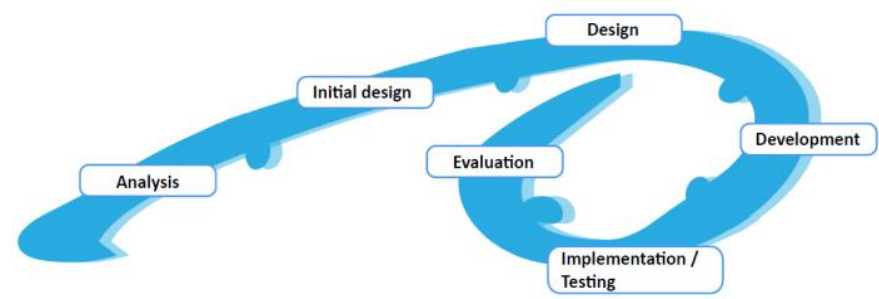

Fig 3 Development Process of MASCA Game

\section{Analysis}

The overall development process starts with an analysis about what the game should teach, after which the game is designed and via numerous iterations updated until it fulfils all training requirements.

The first step of the development process started with gathering information necessary for the initial design. Most of this information resulted from a Training Needs Analysis (TNA), such as demographics of the target population, the available training time and the competencies that need to be trained. The rest of the required information came from the user requirements analysis. This information answers questions regarding four entities: (i) the knowledge domain; (ii) the game principles; (iii) the simulation model and (iv) the didactical principles. The target audience of SKYBOARD consisted of the key stakeholders in the airport turnaround process who are responsible for making decisions for their company with regard to dealing with events in their operations, such as lost passengers, de-icing, closed runways etc.

\section{Initial design}

Based upon the TNA a competency profile was developed, with the key competencies including creating a common understanding of the mutual benefits of implementing A-CDM, communication, joint decision making and information sharing. For the complete profile of Skyboard the reader is referred to Zon and colleagues (Zon, Corrigan, McDonald, \& Maij, 2012). The competency profile forms the basis for the learning elements of the game, while the game dynamics form the framework and the basis for the 'fun' elements of the game. They comprise the way players interact with the game. Examples of game mechanics are the pieces that chess players use to express their moves on the board to the other player, or rolling a dice to determine the likelihood that something happens during game play. Learning elements from the competency profile and 'fun' elements from the game dynamics are necessary to create an effective serious game. 


\section{Design}

Competencies and game dynamics are selected and deselected in this phase of game development to create the best fit between the two. Some game mechanics cover multiple competencies and some none. Competencies that are similar or fit into the same mechanics can be chunked, but care needs to be taken to ensure that players will be rewarded for demonstrating the behaviour that matches with the competency profile and 'punishes' non-compliant behaviour.

During game development, the profile was walked through several times to identify which competencies were inherent to the selected game dynamics. For example, most games require players to continuously assess what the current game state is. This means that players need to understand what has happened and what this means for the situation to be able to decide on their next moves. This assessment of the situation is one of the main competencies that were identified for change managers. Thus, assessing the situation is a competency that is inherent to most games. After identifying the inherent competencies a selection was made of which competencies the game would focus on. The selected competences were mainly soft skills that were highlighted as important for change managers: communication, cooperation, decision-making, planning, implementation and ensuring a positive attitude towards A-CDM.

\section{Develop, test and evaluate}

After this important analysis and initial design phases, the iterative phase started. Part of this phase is aimed at balancing and testplaying the game with prototypes. Balancing is necessary to ensure that each player has an equal likelihood of winning. In serious games design it also entails ensuring that appropriate use of the to-be-trained competencies is more rewarding. An automated procedure based upon a computer model of the game was developed for parts of the balancing work. This procedure was alternated with test playing with real players to compare computer models with the creative and erratic behaviour of real human players.

\section{PLAYING SKYBOARD}

SKYBOARD is a board game that is played by 4 players. Each player represents a stakeholder in the A-CDM process: ATC, Airline, Ground Handling and Airport Operations.

The players aim to get all aircraft to depart on time. However, during the game several bottlenecks occur at random, such as missing passengers, snow on the runways, etc. These bottlenecks make it difficult to adhere to the schedule and forces players to decide which aircraft should be serviced first or maybe be cancelled.

The game can be played in two different modes. In the A-CDM mode players are rewarded and elicited to collaborate and play as they would in an airport where all stakeholders are fully operating according to the A-CDM principles. In the non-A-CDM mode players are encouraged to work as they normally do without a strong necessity to collaborate or work according to A-CDM principles. Based upon the scores that players will accomplish when playing the game and based upon the feedback that players will receive from the trainer the difference between A-CDM and non-A-CDM will become unmistakably clear to all players.

The game board (see figure 4) can be seen as a shared Situational Awareness between the players. The board presents a bird's eye view over the airport. All aircraft standing at the gate, all bottlenecks that need to be solved and the status of every player can be seen. All players are able to see for themselves what the situation at the airport is, where help is needed, where deviations from the planning are taking place. They can decide for themselves whether they should take action and support the other stakeholders or not based upon this knowledge. 


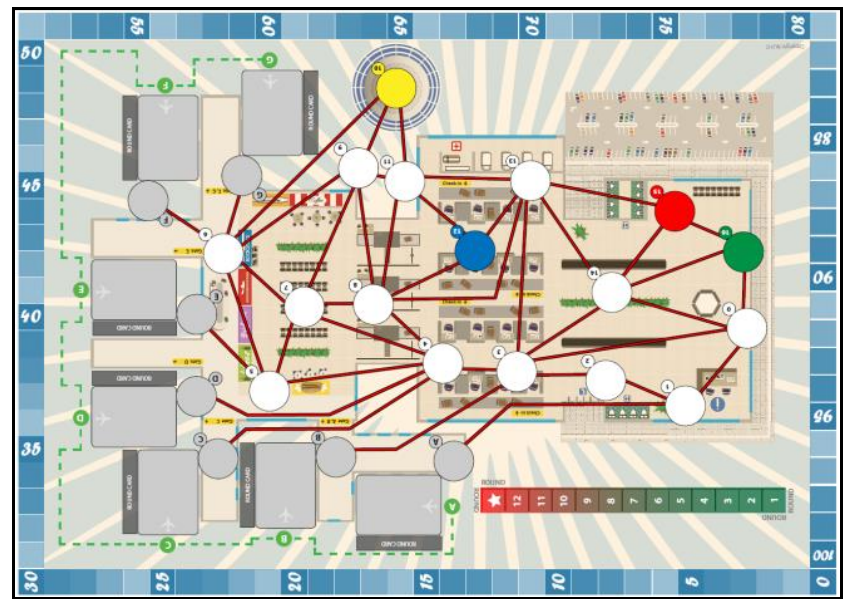

Fig 4 The Game Board, as how it is used in the game. Notice the coloured home bases for all players, the seven gates, and the white circles representing the locations where bottlenecks that disturb the normal airport routine will appear during gameplay

The game can be played without an instructor present. However, a well-trained instructor will enhance training effectiveness. The instructor's task is to observe players during the game to identify behavioural markers that are related to the selected competencies. Upon identifying one of these behaviours, the instructor pauses the game and discusses the behaviour with the players. This way, the players are given the chance to practice the correct behaviours during the same game in a safe learning environment.

\section{RESULTS}

The test sessions during the first development cycles took place within the development team, with gaming and training experts at the Dutch National Aerospace Laboratory - NLR and with training experts at Trinity College Dublin. Three more larger test sessions /workshops were held at the airport with representatives of the target group.

Each of the large sessions was preceded by a presentation of the role of the game in the process of introduction of A-CDM and test play to familiarise the participants with the new game. Instructors were present during the test sessions, but their primary role was to guide participants through the game rules and to gather information on improvements. Therefore, feedback on the competencies was not provided by the instructors during gameplay.

Before and after playing the game, participants were asked to fill out a questionnaire aimed at identifying their attitudes towards ACDM and Serious Games in general and towards SKYBOARD specifically. The following paragraphs present these test sessions and the results they have generated.

A final validation test has recently been undertaken to identify if participants learned the competencies the game aims for and if participants enjoy playing the game. The results of this validation will be published shortly.

\section{First Test Session}

The first of these sessions was intended to test the concept of Serious Games and to generate ideas for further development. An early prototype of the game was played and commented on by airport staff. This session focused on game dynamics and on how representatives of the target group considered serious games. Fourteen persons participated in this test session. All participants were employed by the airport were A-CDM was being introduced.

The participants were very enthusiastic about using a Serious Game to aid the introduction of A-CDM. They were positive about physically meeting other representatives of airport stakeholders, instead of only talking on the phone or emailing. However, the game dynamics were not up to the necessary standard. The game did invite participants to discuss A-CDM related issues with other participants, but it did not explicitly encourage them to collaborate. Therefore, the game development after this session focused on developing game dynamics that forced participants to collaborate. A game dynamic that changed after this session was, for example, the introduction of barriers that make it harder for players to achieve their goals individually, while they still could achieve them by collaborating with others. 


\section{Second Test Session}

The target group of the second session consisted of change managers from a number of key stakeholder organisations within the same airport. The goals of this session were to verify how the participants appreciated the improvements that were made to the game and to explore what the participants learned by playing the game. Eight persons participated in this test session. Besides airport employees, also employees from airlines, and ground handlers participated. There was a small overlap, of approximately three persons, with the first session.

This session started with exploring the attitudes of participants towards the introduction of A-CDM. Even though the expectation was that some participants would still be a little unsure of the implementation of A- CDM at the airport all participants indicated that they were looking forward to it. Their expectations for A-CDM were that it would contribute to a better coordination and better predictability of arrival and departure times.

The second research question pertained to the belief of participants that the Serious Game can effectively contribute to learning. Participants were asked to rate several learning environments on their suitability for training skills. After playing the game, the participants were more convinced of the effectiveness of Serious Games compared to their initial attitude towards Serious Games before playing the game (the belief that serious games can be used for training skills and attitudes increased significantly after playing the game $(t(11)=3,, p<0,05)$.

Thirdly, participants were asked which learning goals they thought the game would achieve. Most of them indicated that the main learning goal is in the area of understanding the critical importance of collaboration.

A final research question was to find out how much participants enjoyed playing SKYBOARD. This is an important question, because participants (as stated above) who enjoy a learning experience are more motivated to perform their best and will therefore learn more from their experience. The participants indicated that they enjoyed playing the game. Figure 6 shows the attitudes of participants regarding Skyboard. Participants were asked to place a cross in the questionnaire at the position that they found most appropriate. Afterwards the location of the crosses was measured with a measurement rod, averages were calculated and those were visualised in Figure 6. Participants were satisfied with most aspects of the game, but somewhat less positive on learning how to play the complex game.

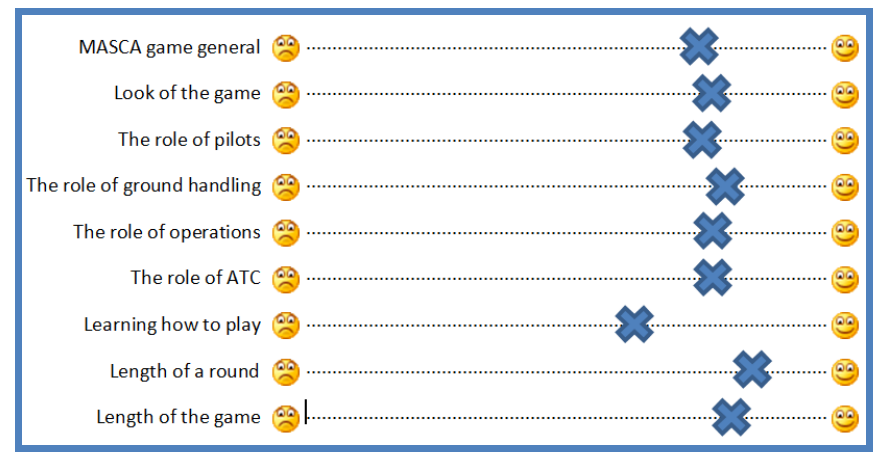

Fig 6 Target Group Attitudes towards Skyboard. Notice that in general people appreciate the game and that the most important aspect to improve is the instructions / tutorial for the game.

\section{Third Test Session}

The third session was performed with the same target group as the second session and it mostly studied the same questions, but it was performed with representatives of other stakeholders from the airport. An additional goal of this session was to study if the game required further improvements or if it was ready for finalisation and validation. Eighteen persons participated in this test session. Besides airport and airline staff also a catering dispatcher, ground handlers, training experts, a fuel company representative and the researchers participated.

The results are mostly comparable to the second test session, though no statistical comparison between both sessions was performed. All participants looked forward to having A-CDM introduced at their airport. They expect better predictability and more accurate information on arrival and departure times. The participants' beliefs towards the effectiveness did not change after playing Skyboard, but were quite positive (7 positive against 1 negative) beforehand. The learning goals that they expected were a better understanding of A-CDM and were in the area of cooperation. 
An important finding from this session was that there were no significant differences in the appreciation of SKYBOARD (F $=.192$; $\mathrm{p}=.977$ ). Thus, the participants in the final test session were comparably satisfied with the game (incl. instructions), indicating that further improvements on game dynamics were no longer necessary and the game was ready for validation.

As a result of the positive aspects of SKYBOARD and the role it played in facilitating more collaborative and co-operative behaviours, the next phase of the overall approach to implementing A-CDM within the airport is to more fully embed the Serious Game into a more specific program focusing on enhancing communication and co-operation among the key stakeholders. This next phase of the learning programme will bring in a wider number of key airport stakeholders emphasising the collaborative learning approach involving groups of learners working together as a team to find a solution and working together in implementing that solution.

\section{CONCLUSIONS}

While the fun element is a key aspect of the Serious Game development, this study and other studies have shown that Serious Games do play a role in fostering the development and improvement of various soft skills, like communication, collaboration or negotiation and to enhance overall collaborative learning (Prensky, 2003; Mansour \& El-Said, 2008). The key benefits of introducing the Serious Game to support the implementation of A-CDM in this case included the opportunity for the key stakeholders to spend significant time with each other, getting to know each other in a fairly relaxed and 'fun' environment and getting a better understanding of each other's roles and perspectives on the turn-around processes at the airport and the challenges they were facing with the implementation of A-CDM. A second benefit of the game was that it raised more awareness and initiated a more in-depth discussion of the implementation of A-CDM and what it meant for each of the stakeholders.

The development, implementation and evaluation of the SKYBOARD Serious Game has demonstrated that serious games can support collaborative learning and that such games should be a key resource in any learning environment. The development of SKYBOARD within the MASCA approach and its integration into a wider training programme will continue to support the implementation of A-CDM at the airport.

One of the most important drivers of the overall MASCA learning process which is largely absent from traditional approaches to training and learning is the process of social interaction and social cohesion. This should create a sense of common purpose that provides a compelling rationale for enhanced collaboration between departments and organisations (the converse of seeing these a separate 'silos'). Therefore what the MASCA learning process advocates is that the social basis of change initiatives need to have some of the characteristics of a team and fosters trust between members and with those directly or indirectly involved with the change; thus, that there is positive constructive engagement rather than alienation. This then provides a basis for establishing an understanding of this common and compatible process and the content of knowledge, competence and capability to achieve the overall strategic plan for change. This level of understanding and learning is vital for the success on implementing A-CDM (and other change initiatives) and ensuring on-going learning.

It is becoming apparent that we are at the beginning of a fundamental shift in the way that both learning and working is happening in organisations. Therefore the establishment of a collaborative learning process and integrated learning package needs to focus on supporting continuous performance improvement and learning (competency and capability at all levels) and to ensure this overall learning is fully aligned to the overall strategic blueprint of the organisation.

A key aspect of this framework is that it requires persuading organisations to this new way of learning. This means recognising it is no longer just about using traditional "command and control" approaches (that are employed in most training solutions to try and force people to learn), but will also involve encouraging and supporting people to engage in new collaborative activities to support one another as they work by helping them to "connect and collaborate" to improve overall performance and ensure longer term sustainability.

\section{ACKNOWLEDGEMENTS}

The above mentioned research has received funding from the European Commission's Seventh Framework Programme FP7/20072013 under grant agreement 266423 "MASCA". 


\section{REFERENCES}

Abt, C. (1970). Serious Games. New York: The Viking Press.

ADAMS, 1994-1999. Human factors in aircraft dispatch and maintenance. Under the EU BRITE-URAM Programme. Contract No. BRPR-T95-0038, Project No. BE95-1732. Brussels: European Commission.

ADAMS II, 2001-2004. Human factors in aircraft dispatch and maintenance. Under the EU BRITE-URAM III Programme for Industrial Materials and Technology. Project No. GRD1-2000-25751. Brussels: European Commission.

Annetta, L. A., Minogue, J., Holmes, S. Y., \& Cheng, M. (2009). Investigating the impact of video games on high school students' engagement and learning about genetics. Computers \& Education, 53, 74-85.

Belanich, J., Orvis, K., \& Sibley, D. (2013). PC-Based Game Features That Influence Instruction and Learner Motivation. Military Psychology 2013, 25(3), 206-217. doi:10.1037/h0094963

Coller, B. D., \& Scott, M. J. (2009). Effectiveness of using a video game to teach a course in mechanical engineering. Computers \& Education, 53, 900-912.

Connolly, T. M., Boyle, E. A., MacArthur, E., Hainey, T., \& Boyle, J. M. (2012). A systematic literature review of empirical evidence on computer games and serious games. Computers \& Education 59, 661-686.

Garris, R., Ahlers, R., \& Driskell, J. E. (2002). Games, motivation, and learning: A research and practice model. Simulation \& Gaming, 33(4), 441-467.

Hart, J. (2012). Social Learning to Work-Force Collaboration. http://www.slideshare.net/janehart/social-collaborative-

learning-in-the-workplace

Johnsson, M., Boud, D., \& Solomon, N, (2012). Learning in-between, across and beyond workplace boundaries. Special Issue of International Journal of Human Resources Development \& Management on the Boundaries of Workplace Learning, 2012 Vol.12, No.1/2 pp.61 - 76 .

HILAS, 2005-2009. Human integration into the lifecycle of aviation systems. Priority No. 1.4 - Aeronautics and Space Research Area 3. Priority Title: Improving Aircraft Safety and Security, IP8. Proposal/Contract No. 516181. Brussels: European Commission.

Kolb, A., \& Kolb, D. (2005). Learning styles and learning spaces: Enhancing experiential learning in higher education. Academy of Management Learning \& Education, 4(2), 193-212.

Mansour, S., \& El-Said, M. (2008). Multi-player role playing educational serious games: a link between fun and learning. The International Journal of Learning, 15(11), 229-240.

Michael, D. and Chen, S. (2006) Serious Games: Games That Educate, Train and Inform. Boston: Thomson.

Plass, J., O'Keefe, P., Homer, B., Case, J., Hayward, E., Stein, M., \& Perlin, K. (2013). The impact of individual, competitive, and collaborative mathematics game play on learning, performance, and motivation. Journal of Educational Psychology, 105(4), 1050-1066. doi:10.1037/a0032688

Prensky, M. (2003). Digital Game-Based Learning. Computer Entertainment, 1(1), 21-21.

Ritterfeld, U., Shen, C., Wang, H., Nocera, L., \& Ling Wong, W. (2009). Multimodality and interactivity: Connecting properties of serious games with educational outcomes. CyberPsychology \& Behavior, 12(6), 691-697.

Tynjälä, P. (2008). Perspectives into learning at the workplace. Educational Research Review, 3(2), 130-154.

Zon, G. D., Corrigan, S., McDonald, N., \& Maij, A. (2012). A learning, training \& mentoring framework (LTM) \& the role of serious games to facilitate sustainable change in the aviation industry. PSAM-11 ESREL 2012. Helsinki, Finland.

Zyda, M. (2005) From Visual Simulation to Virtual Reality to Games. Computer, 38 (9), p.25-32. 